In der Rubrik „Literatur kompakt" werden die wichtigsten Originalarbeiten aus der internationalen Fachliteratur referiert.

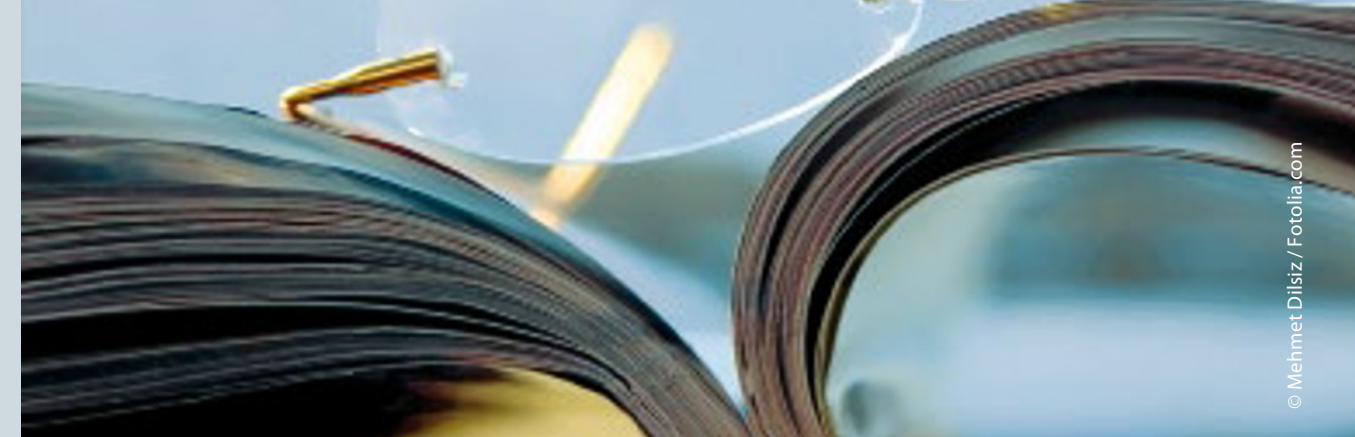

\section{Nach Implantation einer Hüft-TEP verliert der proximale Femur an Knochenmasse}

\begin{abstract}
Benötigen junge und aktive Patienten einen Hüftgelenkersatz, stellt sich die Frage, mit welchem Prothesentyp innen am besten gedient ist. Eine US-amerikanische Studie bestätigt, dass nicht zementierte totale Endoprothesen zum Verlust von Knochenmasse führen.
\end{abstract}

D as Team um Denis Nam von der Washington University School of Medicine hat im Zuge einer Studie über fünf Jahre hinweg den Verlauf nach einem Ersatz des Hüftgelenks bei 96 Pati-

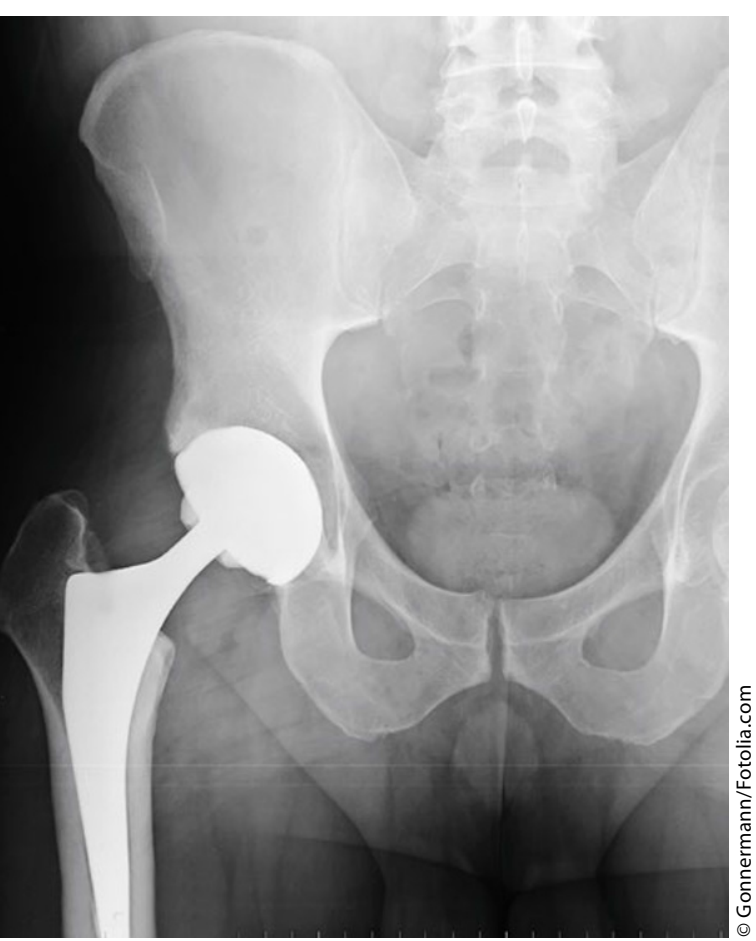

Hier erscheinen die Knochenstrukuren nach der Endoprothesen-Op. unauffällig. enten mit 103 operierten Hüftgelenken verfolgt. 58 Hüftgelenke waren mit einem Oberflächenersatz (OE) versorgt, in 45 Fällen war eine Totalendoprothese (TEP) im zementfreien Verfahren eingebaut worden.

Die Patienten waren im Mittel um die 50 Jahre alt und körperlich aktiv. Im UCLA Activity Score kamen sie auf mindestens sechs von zehn Punkten, was eine regelmäßige Teilnahme an zumindest mäßig belastenden Aktivitäten bedeutet. Im Mittel lagen die Werte bei sieben Punkten. Nach der Operation stieg die Punktzahl auf durchschnittlich 8, was etwa regelmäßigem Golfen oder Kegeln entspricht. Statistisch bestand zwischen der OE- und der TEP-Gruppe kein Unterschied.

Im Harris Hip Score (HHS), mit dem die durch eine Erkrankung des Hüftgelenks verursachten Alltagsprobleme und beschwerden erfasst werden, waren vor der Operation in beiden Gruppen Werte von im Schnitt 60 Punkten zu verzeichnen - gemäß der HHS-Bewertung ein schlechtes Ergebnis. Nach der TEP-Implantation stiegen die Punktzahlen in beiden Gruppen auf durchschnittlich mehr als 90 und lagen damit im als exzellent eingestuften Bereich. Auch hier gab es keine bedeutsame Differenz zwischen OE und TEP.
Unterschiede ergaben sich über die Zeit hinweg mit Blick auf die Knochendichte im Oberschenkel. Hier zeigte sich nach einem Oberflächenersatz während der Beobachtungszeit kein Rückgang der Knochendichte in den sieben Gruen-Zonen des Femur. Eher war im lateralen Bereich sogar eine Zunahme zu verzeichnen. Hingegen waren nach Einbau einer zementfreien TEP über die Zeit hinweg signifikante Abnahmen der Knochenmineraldichte in den GruenZonen 1, 2 und 7 festzustellen, also lateral am Trochanter major und im darunterliegenden Bereich sowie medial im Gebiet des Trochanter minor. In Zone 7 gab es den stärksten Rückgang, im Calcar femorale fiel der Dichtewert im Vergleich zum Ausgangswert auf 89,2\%.

Fazit: Ein Verlust periprothetischen Knochengewebes nach TEP-Einbau wurde bereits in früheren Studien dokumentiert. Der Verlust wird auf die prothesenbedingte Abschirmung des Knochens gegen Belastung zurückgeführt, er erhöht die Gefahr einer aseptischen Lockerung. Mit den vorliegenden Ergebnissen ist klar, dass der Knochenabbau im proximalen Femur nach TEP auch bei jungen, aktiven Patienten eintritt und langzeitig vonstatten geht. Unklar bleibt, was der Abbau klinisch bedeutet. Nam und Mitarbeiter zufolge könnten die Ergebnisse ihrer Studie jedenfalls helfen, Endoprothesen zu konstruieren, die einen physiologischeren Belastungstransfer im proximalen Femur ermöglichen.

Dr. Robert Bublak

Nam D et al. Proximal Femur Bone Density Decreases Up to Five Years After Total Hip Arthroplasty in Young, Active Patients. J Arthroplasty 2016; doi: 10.1016/j.th.2016.05.059 